\section{(6) OPEN ACCESS}

\title{
Dysregulated bioenergetics: a key regulator of joint inflammation
}

\author{
M Biniecka, ${ }^{1}$ M Canavan, ${ }^{2}$ T McGarry, ${ }^{2}$ W Gao, ${ }^{1}$ J McCormick, ${ }^{1}$ S Cregan, ${ }^{1}$ \\ L Gallagher, ${ }^{1}$ T Smith, ${ }^{1} \mathrm{~J}$ J Phelan, ${ }^{4} \mathrm{~J} \mathrm{Ryan}^{5}{ }^{5} \mathrm{~J} \mathrm{O}^{\prime}$ Sullivan, ${ }^{4} \mathrm{C} \mathrm{T} \mathrm{Ng}{ }^{3} \mathrm{D}$ J Veale, ${ }^{1}$ \\ U Fearon ${ }^{2}$
}

\section{Handling editor Tore K Kvien}

- Additional material is published online only. To view please visit the journal online (http://dx.doi.org/10.1136/ annrheumdis-2015-208476).

${ }^{1}$ Centre for Arthritis and Rheumatic Diseases, Dublin Academic Medical Centre, St. Vincent's University Hospital, Dublin, Ireland ${ }^{2}$ Department of Molecular Rheumatology, Trinity Biomedical Sciences Institute, Trinity College Dublin, Dublin, Ireland

${ }^{3}$ Department of Rheumatology and Immunology, Singapore General Hospital, Singapore, Singapore

${ }^{4}$ Department of Surgery, Trinity College Dublin, St James's Hospital, Dublin, Ireland ${ }^{5}$ Department of Radiology, School of Medicine and Biomedical Sciences, University College Dublin, Dublin, Ireland

\section{Correspondence to} Professor Ursula Fearon, Department of Molecular Rheumatology, Trinity Biomedical Sciences Institute, Trinity College Dublin, Dublin D4, Ireland; fearonu@tcd.ie

$\mathrm{MB}$ and $\mathrm{MC}$ are joint first authors.

Received 26 August 2015 Revised 28 January 2016 Accepted 3 March 2016 Published Online First 24 March 2016

To cite: Biniecka $M$, Canavan M, McGarry T, et al. Ann Rheum Dis 2016;75:2192-2200.

\section{ABSTRACT}

Objectives This study examines the relationship between synovial hypoxia and cellular bioenergetics with synovial inflammation.

Methods Primary rheumatoid arthritis synovial fibroblasts (RASF) were cultured with hypoxia, dimethyloxalylglycine (DMOG) or metabolic intermediates. Mitochondrial respiration, mitochondrial DNA mutations, cell invasion, cytokines, glucose and lactate were quantified using specific functional assays. RASF metabolism was assessed by the XF24-Flux Analyzer. Mitochondrial structural morphology was assessed by transmission electron microscopy (TEM). In vivo synovial tissue oxygen $\left(\mathrm{tpO}_{2} \mathrm{mmHg}\right.$ ) was measured in patients with inflammatory arthritis $(n=42)$ at arthroscopy, and markers of glycolysis/oxidative phosphorylation (glyceraldehyde 3-phosphate dehydrogenase (GAPDH), PKM2, GLUT1, ATP) were quantified by immunohistology. A subgroup of patients underwent contiguous MRI and positron emission tomography (PET)/CT imaging. RASF and human dermal microvascular endothelial cells (HMVEC) migration/ angiogenesis, transcriptional activation (HIF1 $\alpha$, pSTAT3, Notch $1-I C)$ and cytokines were examined in the presence of glycolytic inhibitor 3-(3-Pyridinyl)-1-(4-pyridinyl)-2propen-1-one (3PO).

Results DMOG significantly increased mtDNA mutations, mitochondrial membrane potential, mitochondrial mass, reactive oxygen species and glycolytic RASF activity with concomitant attenuation of mitochondrial respiration and ATP activity (all $p<0.01$ ). This was coupled with altered mitochondrial morphology. Hypoxia-induced lactate levels $(p<0.01)$, which in turn induced basic fibroblast growth factor (bFGF) secretion and RASF invasiveness (all $p<0.05$ ). In vivo glycolytic markers were inversely associated with synovial $\mathrm{tpO}_{2}$ levels $<20 \mathrm{~mm} \mathrm{Hg}$, in contrast ATP was significantly reduced (all $p<0.05$ ). Decrease in GAPDH and GLUT1 was paralleled by an increase in in vivo $\mathrm{tpO}_{2}$ in tumour necrosis factor alpha inhibitor (TNFi) responders. Novel PET/MRI hybrid imaging demonstrated close association between metabolic activity and inflammation. 3PO significantly inhibited RASF invasion/ migration, angiogenic tube formation, secretion of proinflammatory mediators (all $p<0.05$ ), and activation of HIF1 $\alpha$, pSTAT3 and Notch-1IC under normoxic and hypoxic conditions.

Conclusions Hypoxia alters cellular bioenergetics by inducing mitochondrial dysfunction and promoting a switch to glycolysis, supporting abnormal angiogenesis, cellular invasion and pannus formation.

\section{INTRODUCTION}

Hypoxia is a fundamental metabolic change common in many inflammatory diseases. The increased proliferation and rapid activation of immune cells requires a switch in cell metabolism from a resting regulatory state to a highly metabolically active state, in order to maintain energy homoeostasis. ${ }^{1}$ This metabolic shift occurs when oxygen levels are low, limiting the metabolism of pyruvate by the tricarboxylic acid (TCA) cycle in the mitochondria during oxidative phosphorylation. We now know that this metabolic shift occurs in many inflammatory conditions such as rheumatoid arthritis (RA) and colitis, with comorbidities including atherosclerosis, diabetes, psoriasis and obesity. ${ }^{2-4}$ One of the earliest events in synovial inflammation is new vessel formation (angiogenesis), resulting in a self-perpetuating and persistent infiltration of leucocytes, transforming the synovial membrane (SM) into an aggressive tumour-like 'pannus' mediating joint destruction. ${ }^{5-}$ ${ }^{8}$ The architecture of the microvasculature is highly dysregulated, thus efficiency of oxygen supply to the synovium is poor. ${ }^{5-7}$ This results in an hypoxic joint microenvironment in vivo, which is associated with increased synovial inflammation and activation of key proinflammatory signalling mediators including HIF1 $\alpha$, nuclear factor kappa (NFкB), Notch and pStat. ${ }^{7}$ 9-15

Hypoxia represents a potential threat to cell function and survival in inflammation, ${ }^{9-11}$ 16-18 with the cellular demand outpacing the vascular supply leading to a switch in mitochondrial respiration in favour of glycolysis. While glycolysis is far less efficient in terms of ATP production compared with the TCA cycle, it produces ATP more rapidly which is required by the highly proliferating cells. This is consistent with studies showing elevated lactate levels with diminished glucose in RA synovial fluids. ${ }^{19-21}$ Lactic acidosis can alter expression of the p53 tumour suppressor gene, and can inhibit DNA repair thus interfering with apoptotic mechanisms, promoting cell survival. ${ }^{22-24}$ Increased glyceraldehyde-3-phosphate dehydrogenase, glucose-6-phosphate and lactate dehydrogenase activity has been demonstrated in RA synovium. ${ }^{25}$ Furthermore, hypoxia induces glucose transporters GLUT1 and GLUT3 in articular chondrocytes, ${ }^{26}$ and several glycolytic enzymes in cartilage explants. $^{27}$

We have previously shown increased mitochondrial DNA mutation frequency and mitochondrial 
dysfunction in the RA joint, ${ }^{28}$ which is associated with oxidative stress, angiogenesis, proinflammatory cytokines and activation of the NLRP3 inflammasome. ${ }^{29-33}$ Furthermore, in RA, studies have shown increased nicotinamide adenine dinucleotide phosphate (NADPH) oxidase/Nox2 in circulating leucocytes and synovium, ${ }^{34}$ demonstrated altered expression of 6-phosphofructo-2kinase/fructose-2, 6-bisphosphatase 3 (PFKFB3) in naive CD4T cells, ${ }^{35}$ and shown synovial deficiency of cytochrome $\mathrm{C}$ oxidase. $^{32}$ The ability of synovial cells to adapt their metabolism in response to the inflammatory microenvironment suggests dysregulated bioenergetics may be an important regulator in RA pathogenesis. In this study we demonstrate alterations in mitochondrial function in response to hypoxia, which is paralleled by a shift in cellular bioenergetics to glycolysis and induction of proinflammatory mechanisms in vitro and in vivo.

\section{METHODS}

Online supplementary file 1 includes descriptions of the following methodologies: patient recruitment, arthroscopy, tissue oxygen $\left(\mathrm{tpO}_{2}\right)$ measurements, primary cell culture, mitochondrial functional assays, quantification of lactate and glucose, transwell invasion assay, ELISA, positron emission tomography (PET)/MRI hybrid imaging protocol, transmission electron microscopy, immunohistochemistry, western blot, wound repair, angiogenic assays and statistics.

\section{Mitochondrial random mutation capture assay}

To characterise the frequency of random mutations in synovial biopsy samples, we used the mitochondrial random mutation capture assay as described previously. ${ }^{28}$ This quantitative PCR-based approach enables precise determination of mutation frequencies following exhaustive digestion of all wild type (non-mutant) sequences by the restriction enzyme Taq-I. Mitochondrial DNA was extracted using a previously reported protocol. ${ }^{28}$ Following extraction, $10 \mu \mathrm{g}$ of $\mathrm{mtDNA}$ was digested with 100 units of Taq $\alpha$ I restriction enzyme (New England Biolabs), $1 \times$ bovine serum albumin and a Taq $\alpha \mathrm{I}-$ specific digestion buffer $(10 \mathrm{mM}$ Tris $\mathrm{HCl}, 10 \mathrm{mM} \mathrm{MgCl} 2,100 \mathrm{mM} \mathrm{NaCl}$ $(\mathrm{pH} \mathrm{8.4))} \mathrm{for} 10 \mathrm{~h}$, with 100 units of Taq- $\alpha \mathrm{I}$ added to the reaction mixture every hour. PCR amplification was performed in $25 \mu \mathrm{L}$ reaction mixtures containing $12.5 \mu \mathrm{L} 2 \times$ SYBR Green Brilliant Mastermix (Stratagene), $0.1 \mu \mathrm{L}$ uracil DNA glycosylase (New England Biolabs), $0.7 \mu \mathrm{L}$ forward and reverse primers (10 pM/ $\mu \mathrm{L}$; DNA oligonucleotide (IDT)) and $6.7 \mu \mathrm{L} \mathrm{H}_{2} \mathrm{O}$. The samples were amplified using a Roche Light Cycler 480, according to the following protocol; $37^{\circ} \mathrm{C}$ for $10 \mathrm{~min}, 95^{\circ} \mathrm{C}$ for $10 \mathrm{~min}$, followed by 45 cycles of $95^{\circ} \mathrm{C}$ for $15 \mathrm{~s}$ and $60^{\circ} \mathrm{C}$ for $1 \mathrm{~min}$. Samples were kept at $72^{\circ} \mathrm{C}$ for $7 \mathrm{~min}$ and following melting curve analysis, immediately stored at $-80^{\circ} \mathrm{C}$. The primer sequences used were as follows: for mtDNA copy number $5^{\prime}$-ACAGTTTATGTAGCTTACCTCC-3' (forward) and 5'-TTGCTGCGTGCTTGATGCTTGT-3' (reverse); for random mutations 5'-CCTCAACAGTTAAATCAACAAAACTGC-3' (forward) and 5'-GCGCTTACTTTGTAGCCTTCA-3' (reverse).

\section{Oxygen consumption rate and extracellular acidification rate-seahorse technology}

Oxygen consumption rate (OCR) and extracellular acidification rate (ECAR), reflecting oxidative phosphorylation and glycolysis, respectively, were measured before and after treatment with oligomycin $(2 \mu \mathrm{g} / \mathrm{mL}$, Seahorse Biosciences, UK), trifluorocarbonylcyanide phenylhydrazone (FCCP) $(5 \mu \mathrm{M}$, Seahorse Biosciences) and antimycin A ( $2 \mu \mathrm{M}$, Seahorse Biosciences) using the Seahorse XF24-analyser (Seahorse Biosciences). Rheumatoid arthritis synovial fibroblasts (RASF) were seeded at 30000 cells per well in 24-well XF-microplates (Seahorse Biosciences) and allowed to adhere for $24 \mathrm{~h}$. Cells were rinsed with assay medium (unbuffered Dulbecco's Modified Eagle's medium (DMEM) supplemented with $10 \mathrm{mM}$ glucose, $\mathrm{pH} 7.4$ ) before incubation with assay medium for $30 \mathrm{~min}$ at $37^{\circ} \mathrm{C}$ in a non- $\mathrm{CO}_{2}$ incubator. Following incubation cells were stimulated with dimethyloxalylglycine (DMOG), a pan prolyl hydroxylase inhibitor and dimethyl sulfoxide (DMSO) vehicle control for $2 \mathrm{~h}$. DMOG (HIF $1 \alpha$ activator), was used in these experiments to mimic the effect of hypoxia as the Seahorse Analyzer cannot be placed in a hypoxic chamber. Four baseline OCR and ECAR measurements were obtained over 28 min before injection of specific metabolic inhibitors. Moreover, to challenge the metabolic capacity of the RASF, three OCR and ECAR measurements were obtained over 15 min following injection with oligomycin, FCCP and antimy$\operatorname{cin} \mathrm{A}$.

\section{RESULTS \\ DMOG induces mitochondrial dysfunction in primary RASF in vitro}

DMOG significantly increased mtDNA mutation frequency from a mean of $3.5 \times 10^{-5}$ to $6.0 \times 10^{-5}(p=0.008)$ (figure $\left.1 \mathrm{~A}\right)$. DMOG significantly induced reactive oxygen species (ROS) production $(p=0.001)$ (figure $1 \mathrm{~B})$, which was coupled by a significant increase in mitochondrial membrane potential (MMP) $(p=0.03)$ (figure 1C) and mitochondrial mass $(M M)(p=0.03)$ (figure 1D). In contrast DMOG significantly inhibited intracellular ATP in RASF $(\mathrm{p}<0.001)$ (figure 1E).

\section{DMOG induces changes in energy metabolism in primary RASF in vitro}

To further investigate if the increased mitochondrial dysfunction and reduced ATP levels in RASF were due to alterations in energy metabolism, we measured the two major energy pathways, oxidative phosphorylation and glycolysis using the Seahorse XF-Analyzer. DMOG significantly increased baseline glycolysis (ECAR) compared with vehicle control $(p<0.001)$ (figure $1 \mathrm{~F}$ ), in contrast baseline mitochondrial respiration (OCR) was significantly reduced $(p<0.001)$ (figure $1 G)$. The inhibition of OCR was paralleled by a significant reduction in ATP synthesis $(p<0.01)$ (figure $1 \mathrm{H})$, maximal respiration $(\mathrm{p}<0.001)$ (figure $1 \mathrm{I})$ and spare respiratory capacity $(\mathrm{p}<0.001)$ (figure 1J). Online supplementary figure S1A, B demonstrates representative OCR and ECAR bioenergetic profiles before and after injections of oligomycin, FCCP and antimycin A for RASF in the presence and absence of DMOG.

\section{Hypoxia induces lactate secretion in primary RASF in vitro}

To examine if this results in an increase in glycolytic metabolites, we exposed RASF to 3\% hypoxia and measured glucose and lactate levels. Hypoxia decreased glucose levels (figure 2A), and significantly increased lactate production from RASF $(p=0.0025)$ (figure 2B). Furthermore levels of lactate in synovial fluid were significantly elevated when compared with serum $(\mathrm{p}<0.05)$ (see online supplementary figure S1C).

Using transmission electron microscopy we observed regularly oval shaped mitochondrial morphology under normoxic conditions (black arrows) (figure 2C), in contrast to a mixture of irregular and regular shaped mitochondria under hypoxic conditions. RASF exposed to hypoxia showed mitochondria that were less dense and had an elongated shape (red arrows), suggesting alteration in mitochondrial function (figure 2D). 

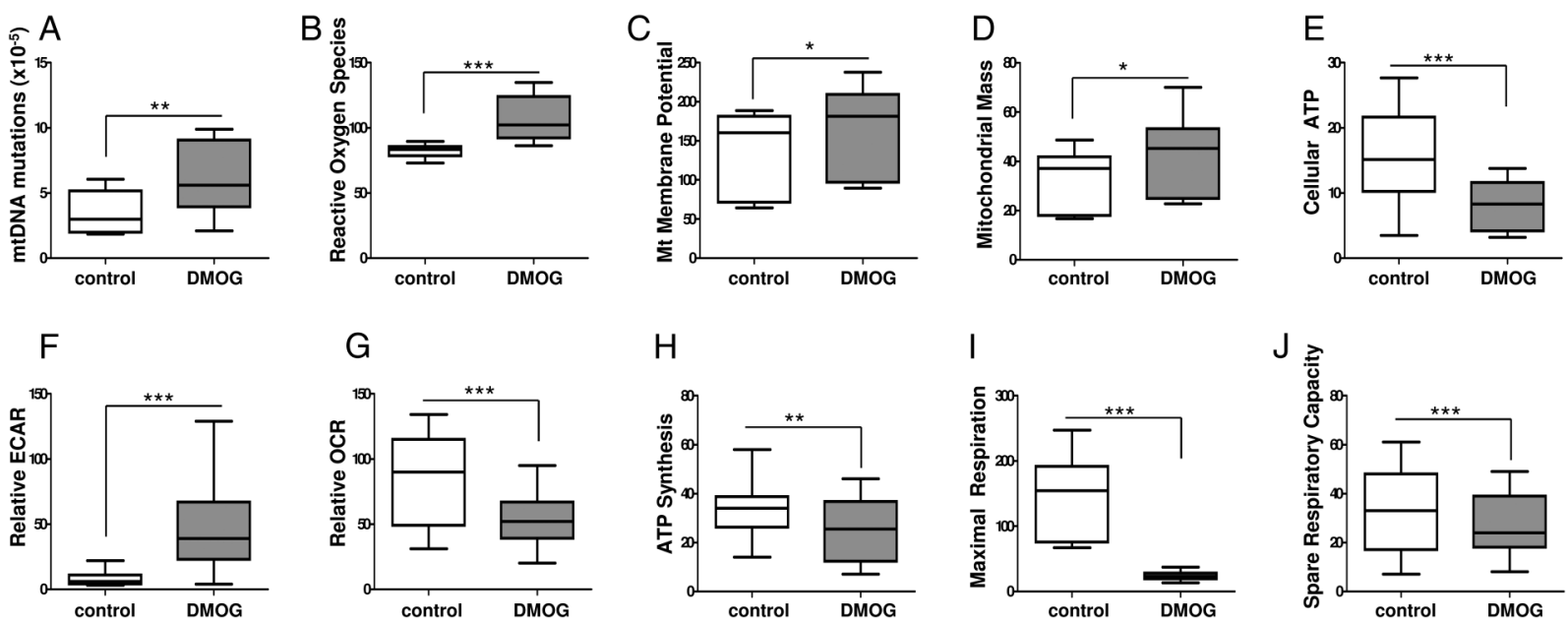

Figure 1 Dimethyloxalylglycine (DMOG) induces mitochondrial dysfunction and glycolytic metabolism in RASF. (A-J) Box plots representing frequency of mitochondrial DNA mutation (A), reactive oxygen species (ROS) (B), mitochondrial membrane potential (MMP) (C), mitochondrial mass $(\mathrm{MM})(\mathrm{D})$, ATP (E) extracellular acidification rates (ECAR) (F), basal oxygen consumption rates (OCR) (G), ATP synthesis (H), maximal respiration (I) and spare respiratory capacity $(\mathrm{J})$, quantification in response to DMOG $(1 \mathrm{mM})$ or vehicle control $(1 \mathrm{mM})$ in primary RASF $(n=7)$. Boxes represent the 25 th to 75 th centiles, lines within the boxes represent the median, and lines outside the boxes represent the 10 th and 90 th centiles. ${ }^{*} p<0.05$ statistically significant.

We then cultured RASF with lactic acid for $24 \mathrm{~h}$ and showed a significant increase in RASF invasion $(p<0.05)$ (figure $2 \mathrm{E}-\mathrm{G}$ ) and basic fibroblast growth factor (bFGF) secretion $(\mathrm{p}<0.05)$ (figure $2 \mathrm{H}$ ), with no effect observed for vascular endothelial growth factor (VEGF), placental growth factor (PIGF) (figure 2I, J), interleukin (IL) 6, IL-8 and RANTES (Regulated on
Activation, Normal T Cell Expressed and Secreted; see online supplementary figure S2A). Pyruvic acid had no effect on IL-6, IL-8 and RANTES (see online supplementary figure S2B), and succinate significantly induced bFGF $(\mathrm{p}<0.05)$ and RASF invasion $(p<0.05)$, similar to that of lactic acid (see online supplementary figure S2C).
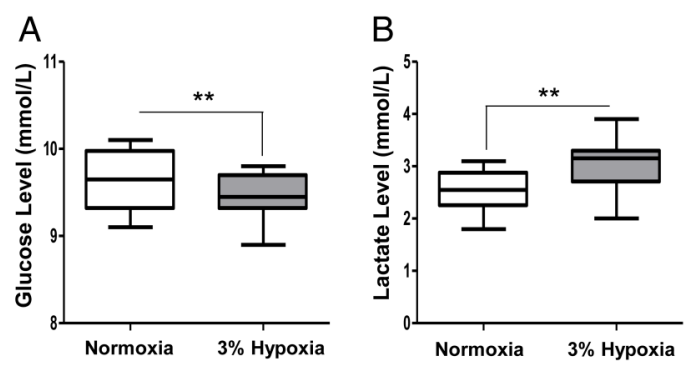

C

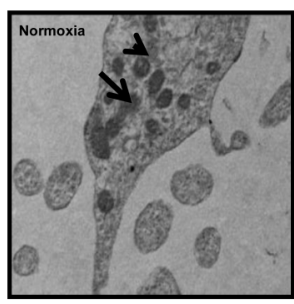

$\mathrm{D}$

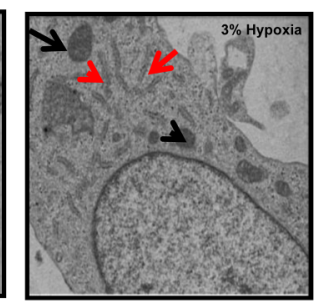

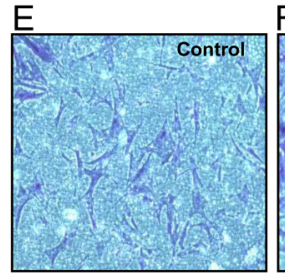

G
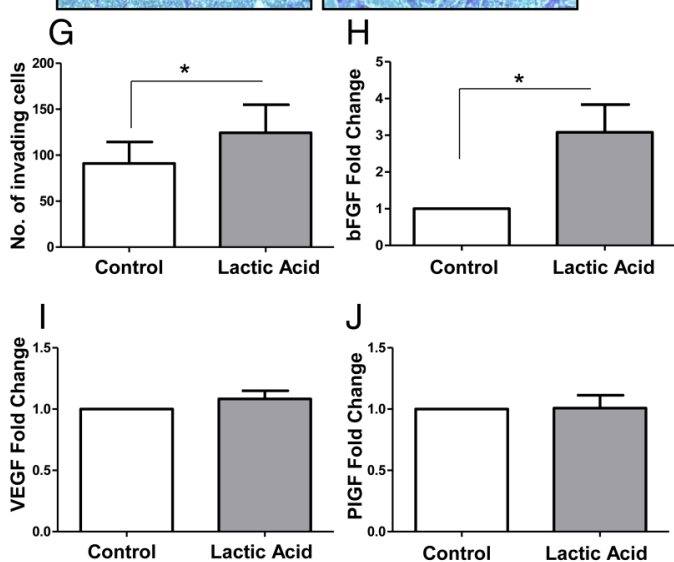

Figure 2 Hypoxia induces lactate, abnormal mitochondrial morphology, invasiveness and secretion of bFGF in RASF in vitro. RASF were cultured under normoxic and $3 \%$ hypoxic conditions for $24 \mathrm{~h}$. Box plots represent glucose $(A)$ and lactate (B) levels in RASF cultured supernatants ( $n=7$ ). Boxes represent the 25th to 75th centiles, lines within the boxes represent the median, and lines outside the boxes represent the 10th and 90th centiles. * $p<0.05$ statistically significant. $(C, D)$ Representative transmission electron microscopy images of RASF mitochondria under normoxic and $3 \%$ hypoxic conditions. Regular shaped mitochondria indicated by black arrows and irregular elongated mitochondria indicated by red arrows. Invasion was assessed using Biocoat Transwell invasion chambers following lactic acid treatment $(1 \mathrm{mM})$ and representative images were taken ( $\mathrm{E}_{\text {, }}$ F). Following $24 \mathrm{~h}$ stimulation, invading cells attached to lower membrane were fixed ( $1 \%$ glutaraldehyde) and stained $(0.1 \% \mathrm{crystal}$ violet) $(\times 40)$. (G) Quantification of RASF invasion ( $n=7)$. Quantification of bFGF (H), VEGF (I) and PIGF (J) in RASF supernatants following culture with lactic acid (1 mM) compared with basal $(n=8)$. * $p<0.05$ statistically significant. 


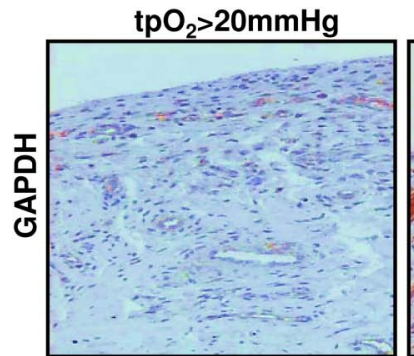

\section{$\mathrm{tpO}_{2}<20 \mathrm{mmHg}$}
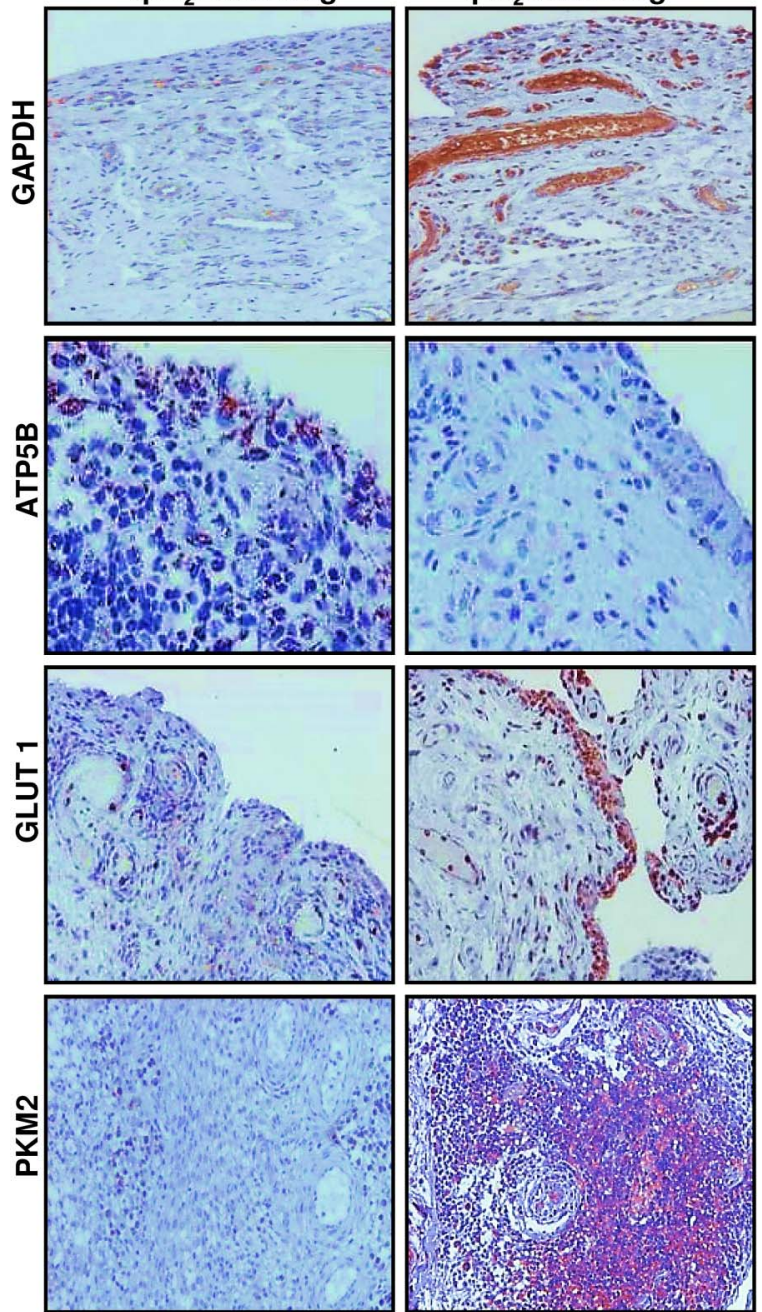
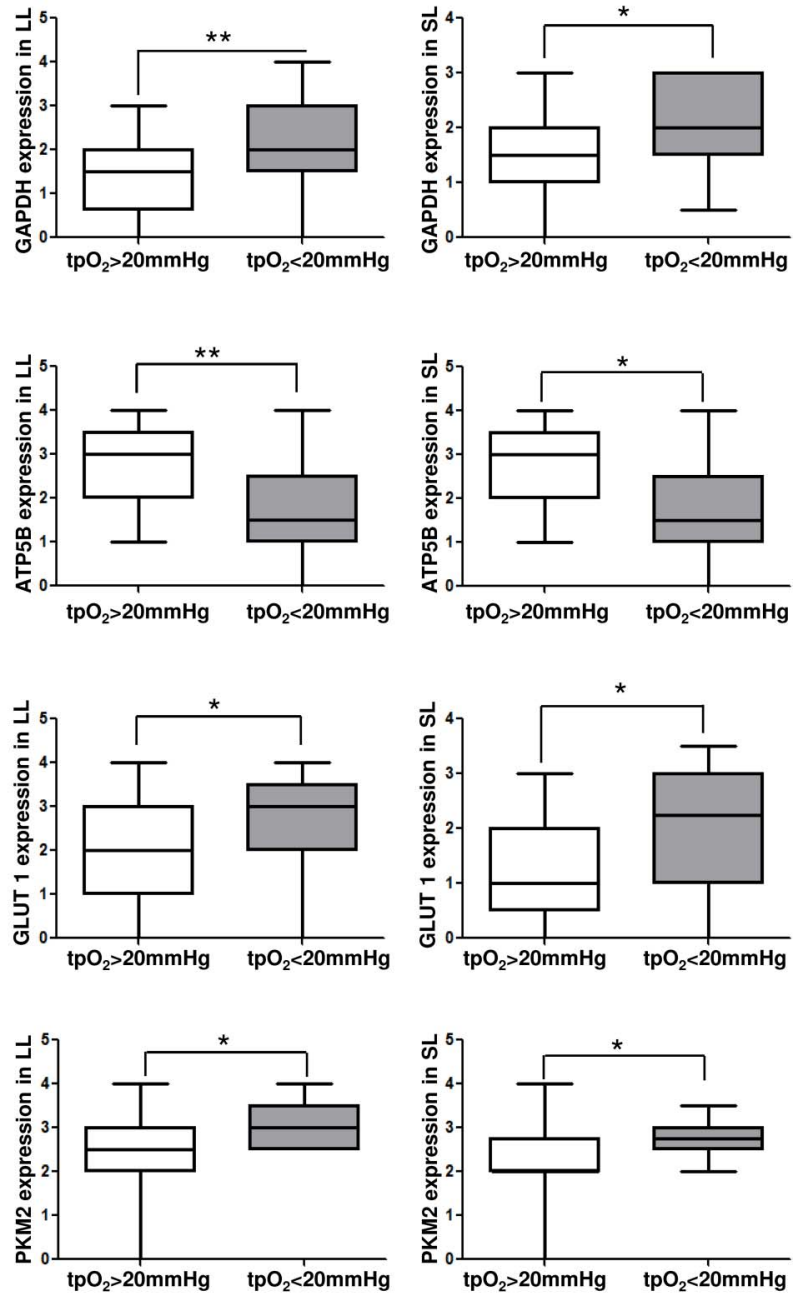

Figure 3 GAPDH, ATP5B, GLUT1 and PKM2 expression in synovial tissue is associated with in vivo synovial $\mathrm{pO}_{2}$ levels. Representative images and semiquantification of immunohistochemical staining for glycolytic and oxidative phosphorylation markers GAPDH, ATP5B, GLUT1 and PKM2 in lining layer (LL) and sublining layer (SL) of synovial tissue. GAPDH, ATP5B, GLUT1 and PKM2 quantification was categorised into patients with in vivo p0 2 levels $>20 \mathrm{~mm} \mathrm{Hg}$ versus $<20 \mathrm{~mm} \mathrm{Hg}$. Quantification was performed using a semiquantitative scoring method (0-4). Boxes represent the 25th to 75th centiles, lines within the boxes represent the median, and lines outside the boxes represent the 10 th and 90 th centiles. ${ }^{*} p<0.05$ statistically significant.

\section{An increase in glycolytic markers with a decrease in ATP is associated with low synovial oxygen partial pressure $\left(\mathrm{pO}_{2}\right)$ levels in vivo}

To confirm a synovial metabolic shift towards glycolysis in vivo, we examined expression of surrogate markers of glycolysis and ATP in synovial tissue by immunohistology in patients $(\mathrm{n}=42)$ whose $\mathrm{pO}_{2}$ levels were quantified. Synovial expression of glyceraldehyde 3-phosphate dehydrogenase (GAPDH) was significantly higher in patients whose $\mathrm{tpO}_{2}$ levels were $<20 \mathrm{~mm} \mathrm{Hg}$ compared with $>20 \mathrm{~mm} \mathrm{Hg}(\mathrm{p}<0.05)$ (figure 3 ). This was in contrast to ATP5B expression which was significantly lower in patients with tpO $\mathrm{p}_{2}<20 \mathrm{~mm} \mathrm{Hg}$ versus those whose $\mathrm{tpO}_{2}$ levels were $>20 \mathrm{~mm} \mathrm{Hg}(\mathrm{p}<0.05)$ (figure 3). Consistent with this finding the glucose transporter GLUT1 and surrogate glycolytic marker PKM2 were also significantly higher in patients with tpO $\mathrm{O}_{2}$ levels in vivo $<20 \mathrm{~mm} \mathrm{Hg}$ versus those whose $\mathrm{tpO}_{2}$ levels were $>20 \mathrm{~mm} \mathrm{Hg}$ (all $\mathrm{p}<0.05$ ) (figure 3). Glycolytic marker expression of GAPDH and PKM2 correlated with increased macroscopic and microscopic vascularity $(\mathrm{r}=0.42, \mathrm{p}=0.042 ; \mathrm{r}=0.45, \mathrm{p}=0.046 ; \mathrm{r}=0.35, \mathrm{p}=0.046$, respectively). Furthermore in vivo $\mathrm{pO}_{2}$ inversely correlated with GAPDH $(\mathrm{r}=-0.45, \mathrm{p}=0.003)$, GLUT1 $(\mathrm{r}=-0.44$, $\mathrm{p}=0.006)$, PKM2 $(\mathrm{r}=-0.5, \mathrm{p}=0.003)$ and positively with ATP $(\mathrm{r}=0.4, \mathrm{p}=0.03)$, and synovitis was associated with GLUT1 expression $(r=0.4, p=0.048)$. This data supports our in vitro data and suggests that alterations in mitochondrial function and energy metabolism regulate inflammation in the RA joint.

A subgroup of patients underwent contiguous PET/CT and MRI $(n=4)$. PET/CT and MRI scans were merged to map metabolic activity to synovial perfusion by accurate anatomical co-location. Figure 4A demonstrates representative images of an axial CT (1), axial PET/CT (2) axial contrast-enhanced MRI (3) and PET/MR merged image (4), where it can clearly be seen that increased inflammation (yellow) is co-localised with metabolic activity (red). Figure 4A, B shows representative hybrid fusion images demonstrating co-localisation of metabolic activity and inflammation and in vivo synovial $\mathrm{pO}_{2}$ levels. 
Figure 4 Co-localisation of metabolic activity and inflammation in vivo using hybrid PET/MRI images. Contiguous

$\mathrm{CT}, \mathrm{PET}$ and MRI scans from patients with rheumatoid arthritis were obtained and then fused to create hybrid PET/MRIs to co-localise metabolic activity and inflammation in the joint. (A) Representative images of axial CT slice (1), axial PET/CT (2), axial contrast-enhanced MRI (3) and hybrid PET/MRI fusion (4). (B) Representative images of four patients demonstrating co-localisation of metabolic activity and inflammation and in vivo synovial $\mathrm{pO}_{2}$ levels.

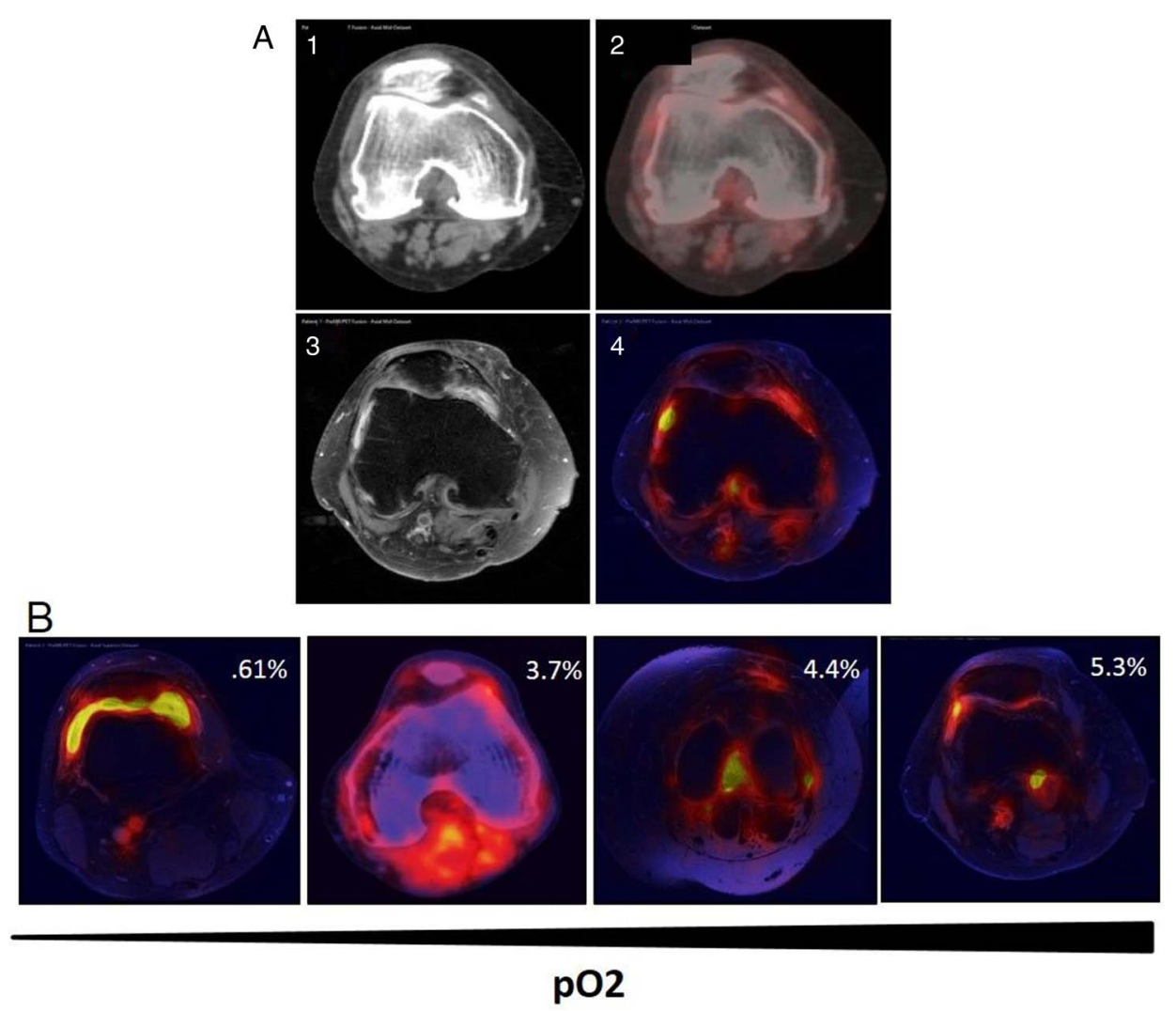

responder versus non-responder patients $(\mathrm{p}<0.05)$ (figure $6 \mathrm{~A}$ ). This was paralleled by a significant decrease in glycolytic enzyme GAPDH and GLUT1 expression in responders only (all $\mathrm{p}<0.05$ ), with no change observed for non-responders (figure 6B-D). Representative images demonstrating GAPDH and GLUT1 pre/post treatment in responder patients who had achieved remission are shown in figure $6 \mathrm{E}, \mathrm{F}$.

\section{DISCUSSION}

In this study, we demonstrate that hypoxia alters cellular bioenergetics by downregulating mitochondrial respiration and promoting glycolysis in the inflamed joint, which in turn induces synovial invasive mechanisms. In primary RASF, we show that DMOG induced higher mitochondrial DNA mutations, MM, MMP and ROS, altered mitochondrial structural morphology, but inhibited ATP. This change was paralleled by an increase in the rate of glycolysis with concomitant attenuation of mitochondrial respiration. Furthermore, we show that hypoxia increased RASF lactate levels, which in turn further induced RASF invasion and bFGF secretion. Increased glycolysis in vitro, was mirrored in vivo, with synovial expression of GAPDH, PKM2 and GLUT1 significantly higher in patients with $\mathrm{tpO}_{2}$ levels in vivo $<20 \mathrm{~mm} \mathrm{Hg}$, in contrast to ATP which was significantly lower, effects of which were reversed in TNFi responders. Finally glycolytic inhibitor 3PO significantly inhibited RASF invasion/ migration, angiogenesis, secretion of proinflammatory mediators and transcriptional activation of HIF1 $\alpha$, pSTAT3 and Notch-1IC.

In this study, we used the mitochondrial random mutation capture (RMC) assay which analyses mitochondrial mutagenesis at single base pair resolution and detects one mutation among 10 million wild type bases. $^{36}$ We demonstrate that DMOG induces random mitochondrial point mutations, paralleled by increases in ROS production, MMP and MM in RASF. The 

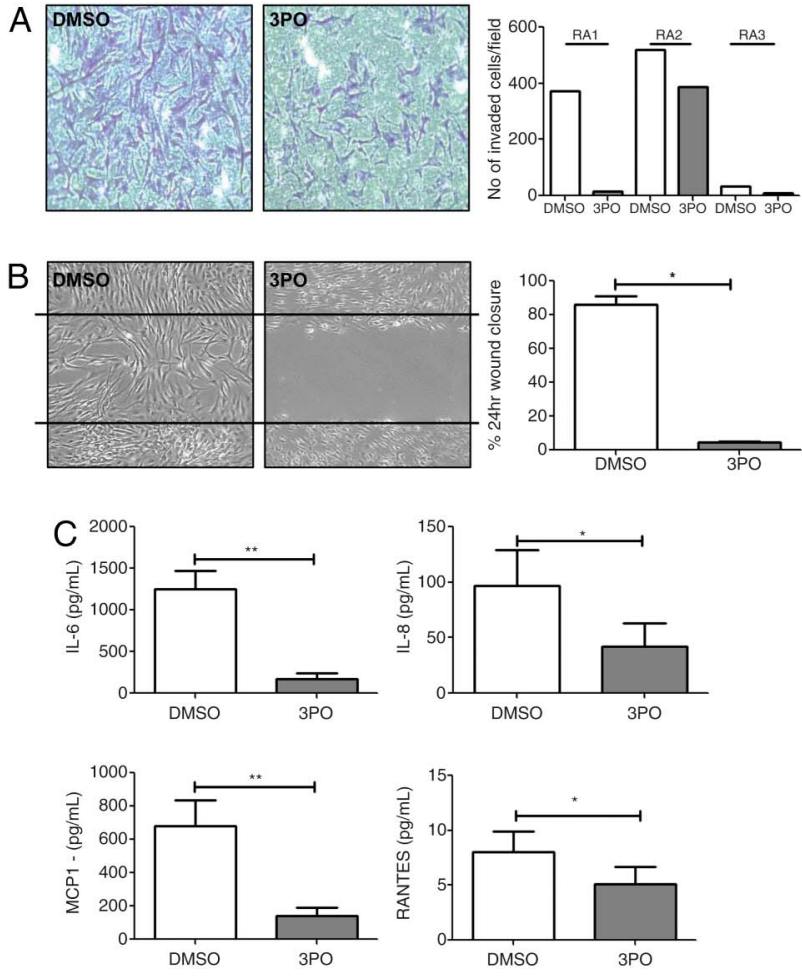

Figure $53 \mathrm{PO}$ inhibits proinflammatory mechanisms in RASF and HMVEC cells. RASF (left panel) and HMVEC (right panel) were cultured in the presence of the glycolytic inhibitor 3PO $(20 \mu \mathrm{M})$. Invasion was assessed using Biocoat Transwell invasion chambers following RASF treatment with 3PO $(n=3)$ and representative images and quantification is shown in (A). Representative images of inhibition of RASF migration following 3PO treatment and quantification of wound repair $(\mathrm{n}=6)$ are shown in (B). (C) Quantification of interleukin (IL) 6, IL-8, MCP-1 and RANTES in RASF supernatants following RASF culture with $3 P 0$ compared with basal $(n=9)$. Representative images and quantification of HMVEC tube formation in response to 3PO (D). Quantification of IL-6, IL-8, GRO and RANTES in HMVEC supernatants following culture with 3PO compared with basal ( $\mathrm{n}=8$ ) (E). Representative western blots are shown in (F) for HIF1 $\alpha$, pSTAT3, tSTAT3, Notch1 and ßactin in both K4IM (left panel) and HMVEC (right panel) following $3 \mathrm{PO}$ treatment under hypoxia. ${ }^{*} \mathrm{p}<0.05$ statistically significant.

mitochondrial genome is highly susceptible to oxidative damage, and implicated in disease progression and response to therapy in inflammatory arthritis. ${ }^{37}$ This is consistent with studies demonstrating increased DNA damage, mtDNA mutation frequency and depolarised mitochondria in RA synovial tissue and peripheral blood mononuclear cells. ${ }^{7} 283038$ In vivo synovial $\mathrm{tpO}_{2}$ levels inversely correlate with mitochondrial mtDNA mutations, 4-hydroxy-2-nonenal (4HNE), 8-oxodG and NADPH oxidase, ${ }^{7} 28303237$ and deficiency of cytochrome C. ${ }^{37}$ Interestingly the effect of DMOG in this study differs from that observed previously using an immortalised healthy synovial fibroblast cell line, where we demonstrated DMOG had no effect on mitochondria function under normoxic conditions. ${ }^{32}$ The difference may suggest that the RASF are more primed and this is an intrinsic effect due to the transformed nature of RASF compared with that of an immortalised cell line. The limited effect under hypoxic conditions is not surprising as the prolyl hydroxylase enzymes are already inhibited, therefore the addition of DMOG would probably not have any further effects. Increased clonal mtDNA mutation frequency in the MT-ND1 gene for mitochondrially encoded nicotinamide adenine dinucleotide (NADH)-dehydrogenase-1 has been detected in RASF, with potential mutation sites in the major histocompatibility complex (MHC) epitope in patients with RA, but not OA. $^{31}$ TNFR1 mutant mouse fibroblasts show evidence of altered mitochondrial function, with increased oxidative capacity, ROS generation and proinflammatory cytokines. ${ }^{31}$
Mitochondria-derived ROS increases NFkB activation through induction of TNF- $\alpha$ cytotoxicity, with subsequent induction of mitochondrial NADPH oxidase. ${ }^{39} 40$ This is consistent with studies describing inflammatogenic features of oxidatively damaged mtDNA, with increased NFkB activity, TNF $\alpha$ production and induced arthritis in animal models. ${ }^{28} 41$

We demonstrate a significant change in energy metabolism within the joint, with decreased intracellular ATP, increased lactate production and an increase in glycolysis with simultaneous attenuation of mitochondrial respiration. This is supported by studies showing increased glycolytic metabolites in RA synovial fluid. ${ }^{19-21}$ Hypoxia and glycolytic metabolites are known to induce many transcriptional factors, such as HIF $1 \alpha$ and $\mathrm{NF} \kappa \mathrm{B}$ which induce angiogenic growth factors, inflammatory cytokines and extracellular membrane components which in turn enhance further glycolytic activity. ${ }^{10-1629} 38$ An increase in the metabolic state towards glycolysis has been shown in proinflammatory cells that are resident in the inflamed joint such as $\mathrm{T}_{\mathrm{H}} 17$ cells, ${ }^{42}$ macrophages and dendritic cells (DC). ${ }^{43}$

In this study we also demonstrate that lactic acid induced RASF invasion and increased secretion of bFGF, with no effect observed for other growth factors and cytokines. While the exact mechanisms involved are unclear, previous studies have shown that lactic acid can induce angiogenic tube formation via NFKB activation, through the monocarboxylate transporter MCT1, ${ }^{44}$ has the ability to stabilise HIF1 $\alpha$ in macrophages, ${ }^{45}$ and can act as a signalling molecule to 
Figure 6 Successful TNFi treatment increases synovial $\mathrm{pO}_{2}$ levels while decreasing expression of GAPDH and GLUT1. Improvements in synovial $\mathrm{pO}_{2}$ levels in patients who responded to TNFi treatment after 3 months compared with non-responders (A). (B-F) Representative images and semiquantification of immunohistochemical staining for glycolytic marker GAPDH in the lining layer (LL) and sublining layer (SL) of synovial tissue and GLUT1 in the SL of synovial tissue. GAPDH and GLUT1 quantification was categorised into patients who were either responders or non-responders to treatment.

Quantification was performed using a semiquantitative scoring method (0-4). Boxes represent the 25th to 75th centiles, lines within the boxes represent the median, and lines outside the boxes represent the 10th and 90 th centiles. ${ }^{*} p<0.05$ statistically significant.
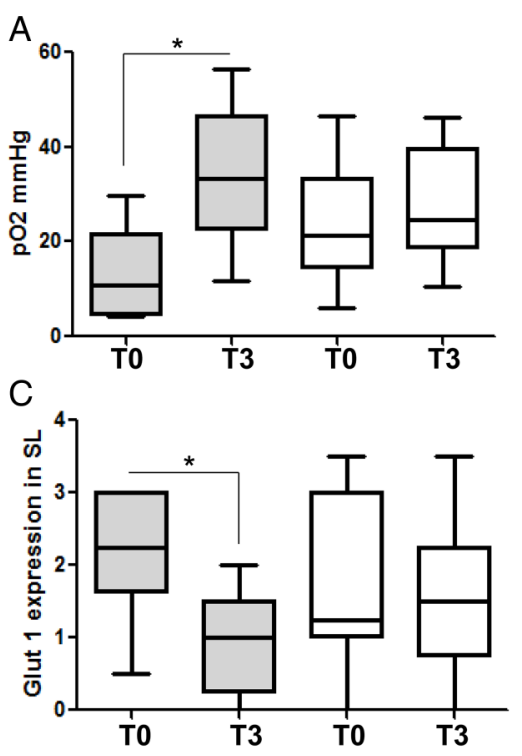

E GLUT 1 in Remission patients

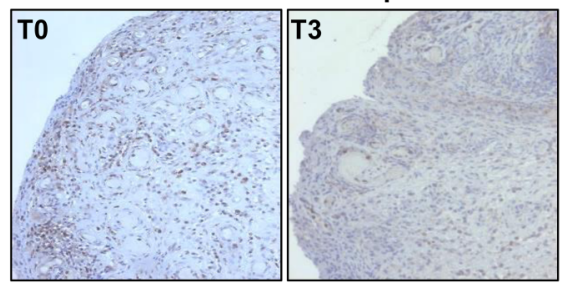

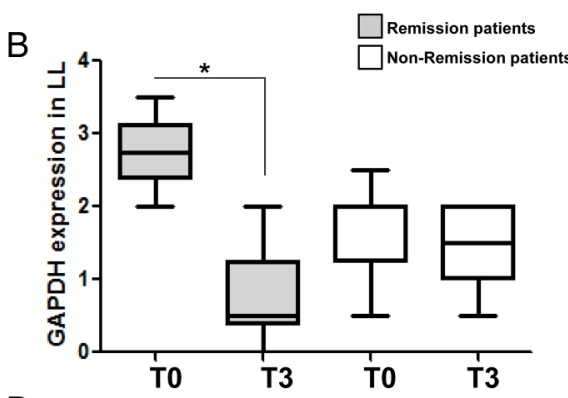

D

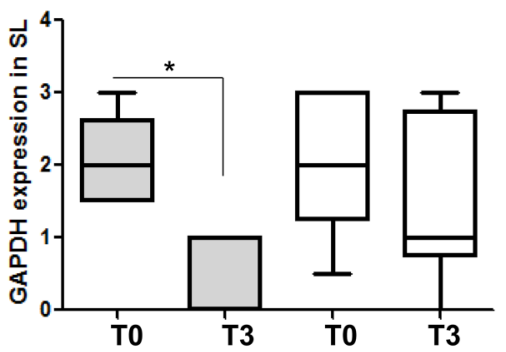

F GAPDH in Remission patients

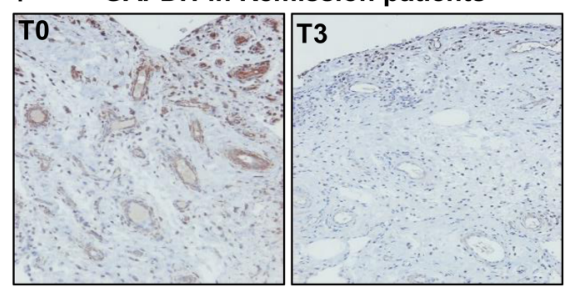

promote cancer cell migration and survival. ${ }^{46}$ Furthermore bFGF itself can further induce dysfunctional angiogenesis, a characteristic feature of the inflamed synovium, ${ }^{6-9}$ can increase cellular invasion, ${ }^{47}$ induce MMP13 in chondrocytes through PI3K/Akt/ERK1/2 pathways ${ }^{48}$ and can activate RhoGTPase proteins, which are pivotally involved promoting cell migration. ${ }^{49}$ Finally, succinate also induced bFGF and invasion, similar to that of lactic acid. The effect of succinate is consistent with other studies showing a marked increase in succinate in lipopolysaccharide (LPS)-activated macrophages, ${ }^{29}$ which in turn inhibits prolyl hydroxylases, resulting in HIF1 $\alpha$ stabilisation, with subsequent induction of proinflammatory mechanisms. Together this data suggests that the shift to glycolysis may be an attempt by synovial cells to produce more ATP rapidly, and can further induce invasive mechanisms.

Our in vitro data is supported by our in vivo data, in which we demonstrate decreased ATP5B in the SM of patients with $\mathrm{tpO}_{2}$ levels $<20 \mathrm{~mm} \mathrm{Hg}$ compared with those $>20 \mathrm{~mm} \mathrm{Hg}$. In parallel, we demonstrated increased expression GAPDH, PKM2 and GLUT1 in low versus high synovial $\mathrm{pO}_{2}$ levels, confirming a glycolytic shift in this hostile environment. This is supported by studies demonstrating lower glucose levels and intracellular ATP in naive CD4 T cells from patients with RA attributed to a deficiency in induction of PFKFB3, a key glycolytic enzyme. ${ }^{35}$ Furthermore it is consistent with a recent study, demonstrating that PKM2 induces HIF1 $\alpha$ and IL-1 $\beta$ in macrophages. ${ }^{50}$ Rescuing mitochondrial defects using antioxidants such as superoxide dismutase (SOD) and $\mathrm{N}$-acetylcysteine (NAC) inhibits the oxidative stress, resulting in inhibition of proinflammatory cytokines, NFkB transcriptional activation and apoptosis. ${ }^{32} 5152$ Several anti-inflammatory drugs such as methotrexate can activate 5' AMP-activated protein kinase $(\mathrm{AMPK})^{53}$ a key regulator of energy metabolism and glucose homoeostasis. Deactivation of
AMPK has been shown to increase mitochondrial dysfunction, whereas activation results in a decrease in activation of $\mathrm{NF} \kappa \mathrm{B}$, HIF $1 \alpha$ and TNF $\alpha$ production. ${ }^{5455}$

To examine if metabolic reprogramming can inhibit proinflammatory mechanisms, cells were treated with $3 \mathrm{PO}$, which is a cell-permeable dipyridinyl-propenone compound that selectively blocks PFK-2 (6-phosphofructo-2-kinase) activity of PFKFB3 isozyme over PFK-1 (6-phosphofructo-1-kinase). 3PO rapidly decreases fructose-2,6-bisphosphate, 2-deoxyglucose uptake and lactate secretion and inhibits cell proliferation and growth. In this study $3 \mathrm{PO}$ inhibited RASF invasion/migration, angiogenesis and proinflammatory cytokines under normoxic and hypoxic conditions. Previous studies examining 3PO showed that PFKFB3 is required for angiogenesis, vessel branching, cell migration and the formation of filopodia/lamellipodia through cytoskeletal rearrangement, ${ }^{56-58}$ however this is the first study to demonstrate the effects of $3 \mathrm{PO}$ on proinflammatory mechanisms in RASF. Furthermore we showed that 3PO differentially inhibited HIF1 $\alpha$, pSTAT3 and Notch-1IC, which have been demonstrated both in vitro and in vivo to be key transcriptional factors involved in mediating hypoxia-induced inflammation in RA. ${ }^{14}{ }^{15}$ Hypoxia is known to potentiate NFкB, Jak-STAT, Notch-1IC signalling via HIF1 $\alpha$ activation and prolyl hydroxylase domain (PHD) suppression, an effect that results in a positive feedback loop on HIF1 $\alpha$ activation. ${ }^{13-16 ~}{ }^{59-61}$ Furthermore we have shown in the RA joint a functional link between HIF1 $\alpha$, STAT3 and Notch-1 signalling in the regulation of proinflammatory mechanisms, ${ }^{14}{ }^{15}$ thus demonstrating complex transcriptional crosstalk within the inflamed joint. Therefore one could hypothesise that reprogramming metabolism with $3 \mathrm{PO}$, could result in reduced accumulation of metabolic intermediates, an increase in oxygen levels, inhibition of transcriptional activation with subsequent resolution of inflammation. 
In summary, we have shown that hypoxia alters cellular bioenergetics by downregulating mitochondrial respiration, promoting a switch to glycolysis in the inflamed joint. This may enable synovial cells to generate sufficient ATP to support enhanced synovial proliferation and pannus formation an effect that can be reversed through metabolic reprogramming.

Twitter Follow Mary Canavan at @mary_canavan

Acknowledgements The authors thank Dr Walsh and Dr Harty for patient recruitment.

Contributors MB, MC, TMCG, WG, JM, SC, LG, TS, CTN, UF, JJP, UF conducted experiments, analysis of data and manuscript preparation. UF, DJV, MB, MC, JOS participated in the data analysis and interpretation and manuscript preparation. UF, DJV, MB, MC and JOS participated in the study design. UF and DJV supervised the research. DJV and CTN recruited all patients, performed the arthroscopies, measured the in vivo $\mathrm{PO}_{2}$ levels and provided all clinical information. JR created MRI/PET hybrid images and designed the imaging programme. DJV, UF, CTN and JR interpreted MRI and PET image data. All authors read and approved the final manuscript.

Funding This work was supported by the Health Research Board of Ireland, the Programme for Research in Third Level Institutions (PRTLI), Ireland and the EU-IM programme 'BeThe Cure' (grant number 4379-2422-R13798).

Competing interests None declared.

Ethics approval St. Vincent's Healthcare Group Medical Research and Ethics Committee.

Provenance and peer review Not commissioned; externally peer reviewed.

Open Access This is an Open Access article distributed in accordance with the Creative Commons Attribution Non Commercial (CC BY-NC 4.0) license, which permits others to distribute, remix, adapt, build upon this work non-commercially, and license their derivative works on different terms, provided the original work is properly cited and the use is non-commercial. See: http://creativecommons.org/ licenses/by-nc/4.0/

\section{REFERENCES}

1 Chang X, Wei C. Glycolysis and rheumatoid arthritis. Int J Rheum Dis 2011;14:217-22

2 Scrivo R, Vasile M, Müller-Ladner U, et al. Rheumatic diseases and obesity: adipocytokines as potential comorbidity biomarkers for cardiovascular diseases. Mediators Inflamm 2013;2013:808125.

3 Choy E, Ganeshalingam K, Semb AG, et al. Cardiovascular risk in rheumatoid arthritis: recent advances in the understanding of the pivotal role of inflammation, risk predictors and the impact of treatment. Rheumatology (Oxford) 2014:53:2143-54.

4 Jiang P, Li H, Li X. Diabetes mellitus risk factors in rheumatoid arthritis: a systematic review and meta-analysis. Clin Exp Rheumatol 2015:33:115-21.

5 Reece RJ, Canete JD, Parsons WJ, et al. Distinct vascular patterns of early synovitis in psoriatic, reactive, and rheumatoid arthritis. Arthritis Rheum 1999;42:1481-4.

6 Szekanecz Z, Koch AE. Endothelial cells in inflammation and angiogenesis. Curr Drug Targets Inflamm Allergy 2005;4:319-23.

7 Kennedy $\mathrm{A}, \mathrm{Ng} \mathrm{CT}$, Biniecka $\mathrm{M}$, et al. Angiogenesis and blood vessel stability in inflammatory arthritis. Arthritis Rheum 2010;62:711-21.

8 Lefèvre $S$, Knedla $A$, Tennie $C$, et al. Synovial fibroblasts spread rheumatoid arthritis to unaffected joints. Nat Med 2009;15:1414-20.

9 Sivakumar B, Akhavani MA, Winlove $C P$, et al. Synovial hypoxia as a cause of tendon rupture in rheumatoid arthritis. J Hand Surg Am 2008;33:49-58.

10 Akhavani MA, Madden L, Buysschaert I, et al. Hypoxia upregulates angiogenesis and synovial cell migration in rheumatoid arthritis. Arthritis Res Ther 2009;11:R64.

$11 \mathrm{Ng} \mathrm{CT}$, Biniecka M, Kennedy A, et al. Synovial tissue hypoxia and inflammation in vivo. Ann Rheum Dis 2010:69:1389-95.

12 Jeon $\mathrm{CH}$, Ahn JK, Chai JY, et al. Hypoxia appears at pre-arthritic stage and shows co-localization with early synovial inflammation in collagen induced arthritis. Clin Exp Rheumatol 2008;26:646-8.

13 Oliver KM, Garvey JF, Ng CT, et al. Hypoxia activates NF-kappaB-dependent gene expression through the canonical signaling pathway. Antioxid Redox Signal 2009:11:2057-64.

14 Gao W, McCormick J, Connolly M, et al. Hypoxia and STAT3 signalling interactions regulate pro-inflammatory pathways in rheumatoid arthritis. Ann Rheum Dis 2015:74:1275-83.

15 Gao W, Sweeney C, Connolly M, et al. Notch-1 mediates hypoxia-induced angiogenesis in rheumatoid arthritis. Arthritis Rheum 2012:64:2104-13.
16 Kammouni W, Wong K, Ma G, et al. Regulation of apoptosis in fibroblast-like synoviocytes by the hypoxia-induced $\mathrm{Bcl}-2$ family member $\mathrm{BCl}$-2/adenovirus $\mathrm{E} 1 \mathrm{~B}$ 19-kd protein-interacting protein 3. Arthritis Rheum 2007;56:2854-63.

17 Gaber T, Schellmann S, Erekul KB, et al. Macrophage migration inhibitory factor counterregulates dexamethasone-mediated suppression of hypoxia-inducible factor- 1 alpha function and differentially influences human CD4+ T cell proliferation under hypoxia. J Immunol 2011;186:764-74

$18 \mathrm{Hu} F, \mathrm{Mu}$ R, Zhu J, et al. Hypoxia and hypoxia-inducible factor- $1 \alpha$ provoke toll-like receptor signalling-induced inflammation in rheumatoid arthritis. Ann Rheum Dis 2014;73:928-36.

19 Naughton D, Whelan M, Smith EC, et al. An investigation of the abnormal metabolic status of synovial fluid from patients with rheumatoid arthritis by high field proton nuclear magnetic resonance spectroscopy. FEBS Lett 1993;317:135-8.

20 Ciurtin C, Cojocaru VM, Miron IM, et al. Correlation between different components of synovial fluid and pathogenesis of rheumatic diseases. Rom J Intern Med 2006;44:171-81.

21 Hitchon CA, El-Gabalawy HS, Bezabeh T. Characterization of synovial tissue from arthritis patients: a proton magnetic resonance spectroscopic investigation. Rheumatol Int 2009:29:1205-11.

22 Firestein GS, Echeverri F, Yeo $M$, et al. Somatic mutations in the p53 tumo suppressor gene in rheumatoid arthritis synovium. Proc Natl Acad Sci USA 1997:94:10895-900.

23 Tak PP, Zvaifler NJ, Green DR, et al. Rheumatoid arthritis and p53: how oxidative stress might alter the course of inflammatory diseases. Immunol Today 2000;21:78-82

24 Gatenby RA, Gawlinski ET. The glycolytic phenotype in carcinogenesis and tumor invasion: insights through mathematical models. Cancer Res 2003;63:3847-54.

25 Henderson B, Bitensky L, Johnstone JJ, et al. Metabolic alterations in human synovial lining cells in pigmented villonodular synovitis. Ann Rheum Dis 1979;38:463-6

26 Peansukmanee S, Vaughan-Thomas A, Carter SD, et al. Effects of hypoxia on glucose transport in primary equine chondrocytes in vitro and evidence of reduced GLUT1 gene expression in pathologic cartilage in vivo. J Orthop Res 2009;27:529-35

27 Ramakrishnan P, Hecht BA, Pedersen DR, et al. Oxidant conditioning protects cartilage from mechanically induced damage. J Orthop Res 2010;28:914-20.

28 Harty LC, Biniecka M, O'Sullivan J, et al. Mitochondrial mutagenesis correlates with the local inflammatory environment in arthritis. Ann Rheum Dis 2012;71:582-8.

29 Tannahill GM, Curtis AM, Adamik J, et al. Succinate is an inflammatory signal that induces IL-1 $\beta$ through HIF-1 $\alpha$. Nature 2013;496:238-42.

30 Biniecka $M$, Kennedy A, Fearon $U$, et al. Oxidative damage in synovial tissue is associated with in vivo hypoxic status in the arthritic joint. Ann Rheum Dis 2010;69:1172-8.

31 Bulua AC, Simon A, Maddipati $R$, et al. Mitochondrial reactive oxygen species promote production of proinflammatory cytokines and are elevated in TNFR1-associated periodic syndrome (TRAPS). J Exp Med 2011;208:519-33.

32 Biniecka M, Fox E, Gao W, et al. Hypoxia induces mitochondrial mutagenesis and dysfunction in inflammatory arthritis. Arthritis Rheum 2011;63:2172-82.

33 Zhou R, Yazdi AS, Menu P, et al. A role for mitochondria in NLRP3 inflammasome activation. Nature 2011;469:221-5.

34 Biniecka M, Connolly M, Gao W, et al. Redox-mediated angiogenesis in the hypoxic joint of inflammatory arthritis. Arthritis Rheumatol 2014;66:3300-10.

35 Yang Z, Fujii H, Mohan SV, et al. Phosphofructokinase deficiency impairs ATP generation, autophagy, and redox balance in rheumatoid arthritis T cells. J Exp Med 2013;210:2119-34.

36 Vermulst M, Bielas JH, Loeb LA. Quantification of random mutations in the mitochondrial genome. Methods 2008;46:263-8

37 Biniecka M, Kennedy A, Ng CT, et al. Successful tumour necrosis factor (TNF) blocking therapy suppresses oxidative stress and hypoxia-induced mitochondrial mutagenesis in inflammatory arthritis. Arthritis Res Ther 2011;13:R121.

38 Moodley D, Mody G, Patel N, et al. Mitochondrial depolarisation and oxidative stress in rheumatoid arthritis patients. Clin Biochem 2008:41:1396-401.

39 Sakon S, Xue X, Takekawa M, et al. NF-kappaB inhibits TNF-induced accumulation of ROS that mediate prolonged MAPK activation and necrotic cell death. EMBO J 2003:22:3898-909.

40 Miesel $\mathrm{R}$, Hartung $\mathrm{R}$, Kroeger $\mathrm{H}$. Priming of NADPH oxidase by tumor necrosis factor alpha in patients with inflammatory and autoimmune rheumatic diseases. Inflammation 1996;20:427-38.

41 Collins LV, Hajizadeh S, Holme E, et al. Endogenously oxidized mitochondrial DNA induces in vivo and in vitro inflammatory responses. J Leukoc Biol 2004;75:995-1000.

42 Shi LZ, Wang R, Huang G, et al. HIF1alpha-dependent glycolytic pathway orchestrates a metabolic checkpoint for the differentiation of TH17 and Treg cells. J Exp Med 2011;208:1367-76.

43 Krawczyk CM, Holowka T, Sun J, et al. Toll-like receptor-induced changes in glycolytic metabolism regulate dendritic cell activation. Blood 2010;115:4742-9.

44 Vegran F, Boidot $\mathrm{R}$, Michiels $\mathrm{C}$, et al. Lactate influx through the endothelial cell monocarboxylate transporter MCT1 supports an NF- $\mathrm{KB} / \mathrm{IL}-8$ pathway that drives tumor angiogenesis. Cancer Res 2011;1:2550-60. 
45 Colegio OR, Chu NQ, Szabo AL, et al. Functional polarization of tumour-associated macrophages by tumour-derived lactic acid. Nature 2014;513:559-63.

46 Gregório I, Martel F, Keating E. Modulation of the uptake of critical nutrients by breast cancer cells by lactate: impact on cell survival, proliferation and migration. Exp Cell Res 2016;341:111-22.

47 Tsuboi R, Sato Y, Rifkin DB. Correlation of Cell Migration, Cell Invasion, Receptor Number, Proteinase Production, and Basic Fibroblast Growth Factor Levels in Endothelial Cells. J Cell Biol 1990;11:511-17.

48 Im HJ, Muddasani P, Natarajan V, et al. Basic fibroblast growth factor stimulates matrix metalloproteinase-13 via the molecular cross-talk between the mitogen-activated protein kinases and protein kinase $\mathrm{C}$ delta pathways in human adult articular chondrocytes. J Biol Chem 2007;282:11110-21.

49 Shin EY, Woo KN, Lee CS, et al. Basic fibroblast growth factor stimulates activation of Rac1 through a p85 betaPIX phosphorylation-dependent pathway. J Biol Chem 2004;279:1994-2004.

50 Palsson-McDermott EM, Curtis AM, Goel G, et al. Pyruvate kinase M2 regulates Hif- $1 \alpha$ activity and IL-1 $\beta$ induction and is a critical determinant of the warburg effect in LPS-activated macrophages. Cell Metab 2015;21:65-80.

51 Iyama S, Okamoto T, Sato T, et al. Treatment of murine collagen-induced arthritis by ex vivo extracellular superoxide dismutase gene transfer. Arthritis Rheum 2001;44:2160-7.

52 Kubota M, Shimmura S, Kubota S, et al. Hydrogen and N-acetyl-L-cysteine rescue oxidative stress-induced angiogenesis in a mouse corneal alkali-burn model. Invest Ophthalmol Vis Sci 2011;52:427-33.
53 Beckers A, Organe S, Timmermans L, et al. Methotrexate enhances the antianabolic and antiproliferative effects of 5-aminoimidazole-4-carboxamide riboside. Mol Cancer Ther 2006;5:2211-17.

54 Yang Z, Kahn BB, Shi $\mathrm{H}$, et al. Macrophage alpha1 AMP-activated protein kinase (alpha1AMPK) antagonizes fatty acid-induced inflammation through SIRT1. J Biol Chem 2010;285:19051-9.

55 Shackelford DB, Vasquez DS, Corbeil J, et al. mTOR and HIF-1alpha-mediated tumor metabolism in an LKB1 mouse model of Peutz-Jeghers syndrome. Proc Natl Acad Sci USA 2009;106:11137-42.

56 Clem B, Telang S, Clem A, et al. Small-molecule inhibition of 6-phosphofructo-2-kinase activity suppresses glycolytic flux and tumor growth. Mol Cancer Ther 2008;7:110-20.

57 De Bock K, Georgiadou M, Schoors $\mathrm{S}$, et al. Role of PFKFB3-driven glycolysis in vessel sprouting. Cell 2013;154:651-63.

58 Schoors S, De Bock K, Cantelmo AR, et al. Partial and transient reduction of glycolysis by PFKFB3 blockade reduces pathological angiogenesis. Cell Metab 2014;19:37-48.

59 Lee JH, Suk J, Park J, et al. Notch signal activates hypoxia pathway through HES1-dependent SRC/signal transducers and activators of transcription 3 pathway. Mol Cancer Res 2009;7:1663-71.

60 Covey MV, Levison SW. Leukemia inhibitory factor participates in the expansion of neural stem/progenitors after perinatal hypoxia/ischemia. Neuroscience 2007;148:501-9.

61 Qiang L, Wu T, Zhang HW, et al. HIF-1 $\alpha$ is critical for hypoxia-mediated maintenance of glioblastoma stem cells by activating Notch signaling pathway. Cell Death Differ 2012;19:284-94. 